- ROBERT C., 2007, «L'impossible "modèle social européen" », Actes de la recherche en sciences sociales, $\mathrm{n}^{\circ} 166-167$, p. $94-109$

Version de l'éditeur disponible sur Internet : [

http://www.cairn.info/resume.php?ID REVUE=ARSS\&ID NUMPUBLIE=ARSS 166\&ID ARTICLE=ARSS $\underline{166[0095]}$

Cécile Robert

CNRS-CERAPS

Université Lille 2, 1 place Déliot

BP629, 59024 Lille Cedex

cecilerobert2@yahoo.com

\title{
L’impossible « modèle social européen »
}

Résumé : Revenant sur la marginalisation et l'illégitimation des enjeux sociaux dans le contexte de l'élargissement, cet article suggère de les mettre en relation avec la position occupée, au sein de la Commission, et plus généralement dans l'espace politique européen, par les services de l'administration communautaire en charge des affaires sociales. Appuyée sur une enquête conduite auprès des agents de ces services, cette recherche interroge plus particulièrement les raisons pour lesquelles ces derniers ont renoncé à défendre l'idée d'un modèle social européen opposable aux pays candidats. Elle s'intéresse dans cette perspective à la manière dont la situation de domination relative de ces services sur les plans institutionnels, matériels et symboliques est perçue et incorporée par leur personnel et oriente leurs modes de mobilisation. A travers l'intériorisation de l'illégitimité de l'Europe sociale, ils contribuent ainsi à son non avènement.

Comment comprendre la place donnée aux questions sociales dans la construction de l'Europe communautaire? Le regard se tourne spontanément vers les arènes intergouvernementales bruxelloises: s'y expriment en effet depuis l'origine du projet européen les oppositions des représentants des Etats membres à la communautarisation de cet enjeu. Ces résistances nationales se sont en outre, au fil des Traités, institutionnalisées dans des règles et des dispositifs - vote à l'unanimité au Conseil, faiblesse des compétences dévolues dans ce domaine à la Commission et au Parlement, nature résiduelle du droit social européen - qui contiennent et contraignent les voies possibles de développement de l'Europe sociale. Pour structurantes qu'elles soient, ces logiques politiques et institutionnelles ne sauraient toutefois épuiser le sens de son non avènement. A travers l'exercice de leur pouvoir d'initiative, et leur travail de préparation des propositions examinées au Conseil, les agents de l'administration communautaire participent également - de manière aussi peu visible qu'essentielle - à définir l'espace des possibles et les contours de l'Europe sociale ${ }^{1}$.

\footnotetext{
${ }^{1}$ Plusieurs études conduites sur les politiques sociales européennes explorent cette hypothèse bien que dans une perspective un peu différente: S. Leibfried et P. Pierson (dir.), Politiques sociales européennes : entre fragmentation et intégration, Paris, L'Harmattan, 1998 ; L. Cram, «Calling the tune without paying the piper ? Social policy regulation : the role of the Commission in EC social policy », Policy and politics, 21 (2), 1993.
} 
C'est notamment le cas des services de la Commission regroupés dans sa direction générale en charge de l'emploi, des relations industrielles et des affaires sociales (DG V) ${ }^{2}$. La DG V demeure pourtant, du point de vue sociologique, un territoire largement méconnu : les effets propres du caractère traditionnellement désincarné (ou, pour le dire autrement, peu centré sur les acteurs, leurs carrières, profils et pratiques) des approches théoriques de l'objet communautaire se redoublent ici du postulat, fondé sur la faiblesse de leurs prérogatives formelles, d'une relative impuissance de ces services. Si les rares enquêtes disponibles soulignent le caractère comparativement limité, par rapport à d'autres segments de la Commission, de leurs ressources institutionnelles, financières et même symboliques ${ }^{3}$, elles laissent le plus souvent sans réponse la question des effets produits par cette situation sur les positions et dispositions de ceux qui y travaillent.

C'est à faire émerger quelques hypothèses exploratoires relatives aux représentations forgées par ces agents de l'Europe sociale et du rôle qu'ils doivent y jouer, et à la manière dont elles orientent leurs modes de mobilisation que s'attache ce papier. A partir d'une enquête conduite dans la direction générale en charge des affaires sociales au moment des négociations d'adhésion avec les pays d'Europe centrale et orientale (voir encadré $n^{\circ} 1$ ), il observe comment la perception et l'incorporation, par ces personnels, de la marginalité de leurs fonctions et domaines d'action dans l'espace politique européen ont pesé sur leur traitement de ce dossier et participé ainsi de l'illégitimation de la question sociale dans le contexte de l'élargissement.

L'enquête se concentre plus spécifiquement sur l'étude d'une controverse, restée interne à la Commission, portant sur la définition de « l'acquis social communautaire » devant être repris par les nouveaux Etats membres. Du milieu à la fin des années quatre-vingt-dix, les services de la Commission européenne ont en effet été chargés de formaliser les modalités des négociations d'adhésion, et en particulier les exigences opposables aux pays candidats. Les agents de la DG V ont été sollicités dans ce cadre, par certains de leurs collègues appartenant à la direction générale en charge des relations extérieures (DG IA), pour revendiquer une acception large de l'acquis social - qui fasse valoir le respect d'un ensemble de principes communs à l'ensemble des systèmes nationaux des Quinze, et notamment l'assurance d'un certain niveau de protection sociale - (voir encadré $n^{\circ} 2$ ). Ils lui ont pourtant préféré une conception plus restrictive, limitée aux seuls règlements et directives existants ${ }^{4}$. A travers l'adoption, par la voix de ces agents, puis dans le cadre de l'adhésion, d'un traitement strictement juridique de la dimension sociale de l'élargissement, ces services ont ainsi contribué à la clôture du débat politique sur ces sujets et in fine à leur non prise en compte.

\footnotetext{
2 Elle est désignée à l'époque, comme les autres directions générales de la Commission, par des chiffres romains aujourd'hui remplacés par les premières lettres du domaine couverts (DG EMPL pour DG emploi), sous le nom de DG V. Nous avons conservé cette appellation pour ce papier.

3 Voir par exemple : M. McDonald, «Identities in the European Commission », in N. Nugent (dir.), At the heart of the Union. Studies of the European Commission, London, Palgrave, (1 ${ }^{\text {ère }}$ éd. 1997); M. Cini, The European Commission. Leadership, organisation and culture in the EU administration, Manchester, Manchester University Press, 1997.

4 Couvrant à l'époque les domaines suivants : l'égalité de traitement entre les hommes et les femmes, en matière d'emploi, de sécurité sociale et de salaires, la non-discrimination en matière de sécurité sociale des travailleurs migrants, la santé et la sécurité au travail et le dialogue social.
} 
Les conditions particulières dans lesquelles s'est déroulée cette enquête ${ }^{5}$ ont largement déterminé la nature des sources exploitées dans ce papier. Les difficultés d'accès au terrain européen mériteraient à elles seules de plus longs développements, soulignant, d'une part, le rapport particulier qu'entretiennent de nombreux hauts fonctionnaires européens au travail sociologique (résistance aux logiques d'objectivation, faible familiarité avec la démarche, crédit symbolique limité reconnu au statut de doctorant ou de chercheur au regard des diplômes revendiqués par ces personnels et du prestige qu'ils accordent à leur propre fonction) et, d'autre part, les inquiétudes couramment suscitées dans cette administration par les demandes d'information venues de l'extérieur (assimilation fréquente des enquêteurs aux lobbyistes et/ou aux journalistes, nécessité, ressentie plus fortement qu'ailleurs, de faire corps par le discours avec l'institution). Ces difficultés sont ici redoublées par les tensions que suscitent dans les services le travail de préparation des négociations d'adhésion : de nature à alimenter les rivalités entre les différents secteurs et segments de la Commission, ce contexte impose dans le même temps le respect de règles de confidentialité plus strictes encore que d'ordinaire. Ces conditions d'enquête ont limité les possibilités d'un travail sociographique systématique sur le personnel de la DG V: elles ont aussi conduit à multiplier les entretiens avec la dizaine de personnes interrogées dans ces services, ce qui leur a parfois conféré une tonalité de confidence et permis surtout l'accès aux archives administratives internes relative au dossier sur l'élargissement. Celles-ci sont particulièrement riches dans la mesure où elles sont constituées d'échanges écrits autour d'un rapport (voir encadré $\mathrm{n}^{\circ} 2$ ), soumis par les partisans d'une définition extensive de l'acquis social communautaire pour correction et approbation aux services de la DG V. L'adoption du rapport par ces services conditionnait en effet la défense d'un acquis social étendu par la voix de la Commission et ultimement dans le cadre officiel des négociations. L'examen des versions successives de ce document, finalement "enterré », et des corrections écrites suggérées par les agents de la DG V permet ainsi de repérer les points saillants de la controverse et les principes de justification qui y sont mobilisés.

L'analyse de cette controverse restée interne aux services met en évidence ce que les positions officielles ne dessinent qu'en creux: les projets écartés, les ambitions devenues impensables, et les coups et tactiques non joués. Elle permet d'explorer les raisons qui ont conduits ces hauts fonctionnaires à renoncer à une définition de l'Europe sociale, à laquelle ils étaient pourtant globalement favorables et qui pouvait conforter de surcroît leurs prérogatives et domaines d'intervention. L'article revient d'abord sur les effets d'homologie entre la place accordée au social dans l'espace politique et institutionnel européen et les positions occupées par les agents qui en ont la charge au sein de l'administration communautaire : il observe que la marginalité de ces derniers semble les inciter à un hyperconformisme institutionnel, incarné ici par le choix d'une définition juridique plutôt que " politique » de l'Europe sociale. Il met ensuite en évidence des logiques d'auto-censure, qui les conduisent à renoncer à certains arguments en faveur de l'Europe sociale, et qui soulignent l'inadéquation des ressources de la $\mathrm{DG} \mathrm{V}$ avec la rhétorique et les modes de sélection des problèmes prévalants au sein de l'administration communautaire. L'article montre enfin comment les débats autour de la légitimité accordée à la notion même de modèle social européen signalent plus généralement une prise de distance de ces eurofonctionnaires avec les ambitions originelles de la Commission concernant l'intervention européenne en matière sociale.

\footnotetext{
5 Celle-ci conduite principalement à Bruxelles entre 1996 et 1998 s'inscrit dans le cadre d'une thèse de science politique soutenue en décembre 2001 sous le titre: «La fabrique de l'action publique communautaire. Le programme Phare (1989-1998) : enjeux et usages d'une politique européenne incertaine ».
} 


\section{Encadré 1 : Le traitement administratif de l'élargissement à l'Est}

L'élargissement de l'Union européenne aux pays d'Europe centrale et orientale (PECO) a pris depuis la Chute du Mur en 1989 la forme d'un processus long et sinueux. La première partie des années 1990 voit ainsi le développement de programmes "d'assistance technique aux réformes 》 dans ces pays (dont le programme Phare créé fin 1989) et la mise en place de coopérations économiques et politiques. A partir du Conseil européen de Copenhague (juin 1993), qui vient confirmer la perspective d'adhésion et propose une première version des "critères d'adhésion" (institutions stables garantissant la démocratie et le respect des droits de l'homme, économie de marché viable, reprise de l'acquis communautaire), les services de la Commission s'engagent dans un travail de nature essentiellement juridique. Celui-ci vise à recenser les normes communautaires et à vérifier, par le biais de rapports réguliers, leur reprise dans les législations des PECO. Au sein de l'administration européenne, ce travail est principalement assuré par la direction générale en charge des relations politiques extérieures (DG IA), qui coordonne ensuite la participation des directions générales sectorielles (dont la DG V) sollicitées pour définir "l'acquis », puis pour réfléchir à «l'impact de l'élargissement» dans leurs domaines de compétences. Appuyé sur une analyse de l'évolution économique, politique et institutionnelle des pays candidats au regard des critères de Copenhague, à laquelle s'ajoute un exercice de prospective sur les conséquences principalement financières de l'élargissement, un document est présenté par la Commission en juillet 1997 ( "l'Agenda 2000 »et les "Avis sur les pays candidats ») sur la base duquel le Conseil européen de Luxembourg (décembre 1997) annonce officiellement l'ouverture des négociations avec six premiers pays. C'est dans le cadre de ces négociations, longues, cantonnées dans les cercles diplomatiques, et très peu publicisées, que sont finalement précisés les conditions et l'ordre dans lesquels ces pays deviendront des Etats membres. Un Traité d'adhésion est signé à Athènes en avril 2003 avec l'Estonie, la Lettonie, la Lituanie, la Hongrie, la Pologne, la République slovaque, la République tchèque, et la Slovénie, auxquelles s'ajoutent Chypre et Malte.

Encadré 2 : Le rapport sur la dimension sociale de l'élargissement : une mobilisation interne à la Commission

C'est en décembre 1995 qu'émerge, au sein des services en charge des pays d'Europe centrale et orientale (PECO) à la direction générale IA (relations extérieures), le projet d'établir un document définissant les objectifs de l'aide communautaire apportée aux pays candidats dans le domaine social et suggérant de promouvoir un modèle social européen dans les négociations d'adhésion. Préparé par la responsable des programmes "sociaux», assistée par une équipe de consultants francobritanniques, le propos du rapport rendu en décembre 1996 s'organise en trois temps : une mise en alerte, soulignant les risques que comporte, pour l'Europe de l'Est et pour l'Union européenne dans la perspective de l'élargissement, le traitement politique réservé à la question sociale dans les PECO ; une mise en accusation, attribuant à l'absence de discours clair, de la part des institutions communautaires, la responsabilité des positions adoptées par les gouvernements est-européens dans ce domaine ; une mise en demeure, invitant l'Union à définir un modèle social européen, qui ne se cantonne pas au seul droit social européen existant mais, rappelle aux pays candidats les grands principes sur lesquels fonctionnent les politiques sociales dans les Etats membres. En même temps qu'il est diffusé informellement auprès de hauts fonctionnaires et responsables politiques nationaux, le document est soumis à l'approbation de la DG V. Une première version de ce document, en date d'octobre 1996, restera un document confidentiel. La seconde, très largement amendée par la DG V, fera l'objet en 1998 d'une diffusion très limitée, et accompagnée de la mention suivante : "Cette étude était financée par le programme Phare de l'Union européenne, DG IA. Les conclusions et interprétations exprimées dans ce document sont seulement celles de [ses auteurs]. Elles ne reflètent pas les politiques de la Commission européenne. " Commission des CE, DGIA (1998), Rapport "Stratégie du secteur social ». Pour une analyse de ce rapport : C. Robert, "Ressources juridiques et stratégies politiques. Analyse d'une controverse à la Commission européenne sur la dimension sociale de l'élargissement de l'Union », Sociologie du Travail, 42 (2), 2000 


\title{
I. Les agents de la DG $V$ au sein de la Commission : positions marginales et postures conformistes
}

\begin{abstract}
«La DGV $V$ c'est une DG faible, à la Commission. Faible parce que c'est un secteur qui a souvent été considéré comme plus faible et puis faible aussi parce qu'ils ne sont pas vraiment très bons. Le problème, c'est que la $D G V$, elle revient toujours avec certaines positions et qu'au bout d'un moment, ça fatigue les gens parce que ça paraît être un peu formel, ça paraît être des slogans. Quand vous avez des gens qui vous disent : 'la priorité c'est de développer des politiques de plein emploi', (soupir). C'est un peu comme le slogan 'la santé pour tous en l'an 2000 ' de l'OMS, ça fait réaliste, hein ?! » (Membre de la DG IA, service " Phare », juin 1997, Bruxelles)
\end{abstract}

Extrait d'un entretien conduit avec un membre d'une autre direction générale, ce commentaire illustre assez justement la position dans laquelle se trouvent placés les agents de la DG V au sein de la Commission, et plus largement dans l'espace politique communautaire. Il met notamment en lumière les liens étroits qui existent entre le degré de communautarisation d'un secteur d'action publique tel qu'il est notamment défini par les Traités successifs - les compétences qu'ils reconnaissent à l'Union dans ce domaine et la manière dont celles-ci sont réparties entre ses différentes entités constituantes -, et les ressources institutionnelles, matérielles, autant que symboliques dont dispose le segment administratif en charge du secteur concerné.

La DG $\mathrm{V}$ semble offrir un exemple emblématique de l'interdépendance de ces ressources. Comme l'évoque la citation précédente, la faible légitimité du social comme secteur d'intervention communautaire, et, partant, les prérogatives limitées et pour le moins controversées de l'administration européenne dans ce domaine se traduisent non seulement par le fait que ses services disposent de moyens juridiques limités par rapport à ceux d'autres directions générales, mais aussi par une difficulté à faire entendre leurs préoccupations, à l'extérieur et au sein même de l'institution. En outre, ainsi que le suggère encore l'extrait d'entretien précédent, ce défaut d'audience est souvent imputé aux compétences et aux postures adoptées par ses agents comme si, par contagion, la relative marginalité de leur domaine d'intervention ne pouvait laisser intacte la réputation de ces personnels. On retrouve ainsi, ça et là, dans les témoignages écrits d'anciens fonctionnaires ${ }^{6}$ ou les rapports de bureaux d'étude7, des allusions aux «dysfonctionnements » et à la "passivité » qui caractériseraient les services en charge des politiques sociales. Dans une enquête conduite pour le compte de la Cellule de prospective, Marc Abélès, Irène Bellier et Maryon Mac Donald soulignent que «dans l'arène européenne, la DG V a rarement eu l'occasion de réclamer un semblant de priorité par rapport aux autres $D G$ et [que] ce manque de considération a suscité une certaine démobilisation des effectifs et un renouvellement relativement important du personnel subalterne dans plusieurs secteurs. (...) Pour les fonctionnaires de la Commission qui se veulent méthodiques, disciplinés, réalistes et rationnels, la DG $\mathrm{V}$ constitue une cible métaphorique facile par opposition à laquelle ils peuvent se définir et à laquelle ils font fréquemment allusion ${ }^{8}$. »

\footnotetext{
${ }^{6}$ H. Michelmann, Organisational effectiveness in a multinational bureaucracy, Farnborough, Saxon House, 1978.

${ }^{7}$ Price Waterhouse, New perspectives for the European Social Fund, Bruxelles, CE, 1990, cité dans A. Smith, L'intégration communautaire face au territoire ..., op. cit.

${ }^{8}$ M. Abélès, I. Bellier, M. Mac Donald, Approche anthropologique de la Commission, Rapport rédigé pour le compte de la Cellule de prospective, décembre 1993, (p.15).
} 
Comme l'indiquent les rares données sociographiques disponibles, la relative marginalité de ces services trouve également à s'incarner dans les profils et carrières de son personnel. L'étude prosopographique réalisée par Marine de Lassalle et Didier Georgakakis montre ainsi que les formations et diplômes de ce personnel - droit/sciences sociales - en font une direction atypique dans une administration où les directeurs généraux revendiquent massivement une double compétence droit/économie. De son côté, Maryon McDonald évoque la présence d'un nombre proportionnellement beaucoup plus important de femmes aux postes de direction (le double de certaines DG) - en lien notamment avec l'appartenance de la direction en charge des questions d'égalité hommes/femmes à la DG V -, participant selon elle de la réputation «d'idéalisme» et de «désorganisation» de ces services ${ }^{9}$. La marginalisation de ces profils est pour partie imputable à des logiques de recrutement spécifiques, qui semblent du reste être remises en question depuis la fin de la dernière décennie $^{10}$. Elle tient également au fait que la hiérarchie symbolique des compétences s'ajuste au prestige respectif dont bénéficie chaque politique sectorielle et le segment administratif qui en a la charge. Les directeurs généraux de la DG $\mathrm{V}$, à l'instar d'autres services en charge de politiques peu investies par l'UE ou encore sous contrôle des Etats membres, font ainsi partie de ceux qui disposent d'un capital institutionnel (spécialisation sectorielle, parcours plus lié aux administrations nationales) plutôt en voie de dépréciation au sein de la Commission ${ }^{11}$.

La spécificité de ces profils contribue à son tour à entretenir l'image d'une direction atypique sinon moins prestigieuse au sein de la Commission. Ainsi, les parcours des agents interrogés laissent penser que ces services, et plus singulièrement encore ceux qui sont en charge des compétences les plus «molles» sur le plan juridique - sécurité et protection sociales, dialogue social - sont des lieux où l'on ne fait pas durablement carrière et qui accueillent plutôt des gens « de passage ». Il s'agit en effet soit de jeunes fonctionnaires qui occupent leur premier poste, soit de personnels qui proviennent des administrations nationales; deux des personnes les plus impliquées dans les discussions autour du rapport ont par ailleurs quitté leurs postes respectifs dans l'année suivante, l'un pour rejoindre une autre direction générale (environnement), l'autre pour un emploi dans un cabinet de conseil parisien, puis dans la haute administration française.

La place occupée par ces agents au sein de l'administration communautaire offre un premier éclairage des postures qu'ils adoptent dans le cadre du débat sur la dimension sociale de l'élargissement. L'hypothèse est en effet permise que la précarité et la marginalité de leurs positions ne sont pas étrangères à la volonté manifestée par ces agents de se conformer d'autant plus rigoureusement aux prescriptions de rôle qu'ils associent au statut de fonctionnaire européen. Telles qu'elles s'expriment à l'occasion de cette controverse, ces références aux devoirs et à l'éthique du fonctionnaire se déclinent autour de deux arguments complémentaires. Les membres de la DG V rappellent d'abord la nécessité dans laquelle ils se

\footnotetext{
${ }^{9}$ M. McDonald, «Identities in the European Commission», in N. Nugent (dir.), At the heart of the Union. Studies of the European Commission, op.cit.

10 Une de nos enquêtes en cours sur la DG5 semble indiquer qu'un tournant a été pris à la fin des années quatrevingt-dix) dans la politique de recrutement, se traduisant par le recours à des profils d'économistes, en particulier pour gérer la «stratégie européenne pour l'emploi » mise en œuvre depuis 1997. De manière significative, les arguments mobilisés soulignent la nécessité de se doter de compétences ad hoc et 'nouvelles' pour la direction générale afin de pouvoir conduire une politique qui se verrait reconnaître une importance - au moins sur le plan symbolique - enfin significative.

11 D. Georgakakis, M. de Lassalle, «Les directeurs généraux de la Commission européenne. Premiers éléments d'une enquête prosopographique ", Regards sociologiques, 27-28, 2004.
} 
trouvent placés de s'ajuster au contexte politique, et en l'occurrence de renoncer à défendre un modèle social européen qui ne saurait faire l'unanimité dans les instances intergouvernementales. Ils soulignent ensuite, avec une égale constance, que sans mandat politique explicite, la seule posture légitime est celle de l'application du droit et de la procédure, à travers l'adoption d'une définition strictement juridique de l'acquis social communautaire

Si la nécessité de penser les conditions de possibilité offertes par le contexte politique est probablement une contrainte largement partagée par les administrations nationales ${ }^{12}$ et internationales, ces préoccupations prennent dans le cadre communautaire une force particulière. Travaillant constamment à donner de la Commission l'image d'un acteur neutre et impartial au service du projet européen, capable de construire le consensus communautaire sur lequel ils font reposer la légitimité de ses politiques, les eurofonctionnaires, plus encore que leurs homologues nationaux, s'efforcent d'esquiver les situations d'affrontement direct avec leurs partenaires au Conseil et au Parlement. Cet impératif du compromis ${ }^{13}$, souvent mis en scène comme une spécificité de l'institution dans les écrits des "pères fondateurs» à travers lesquels s'élabore et se projette l'ethos du fonctionnaire européen ${ }^{14}$, trouve ici son prolongement dans les pratiques et les «manières d'être » revendiquées par les agents de la DG V.

Il s'exprime d'abord sous la forme d'une relecture du passé proche, et plus ancien, du développement communautaire en matière sociale, débouchant sur le constat, formulé sur tous les tons, d'une forme d'impuissance de leurs services à jouer un rôle d'impulsion dans ce domaine. De fait, ces commentaires s'inscrivent dans une histoire jalonnée par les conflits qui ont opposé les Etats membres entre eux et parfois même à la Commission, sur la nécessité et l'intérêt d'un transfert de compétences du niveau national au niveau communautaire. Cette histoire est en outre marquée, depuis le début des années quatre-vingt-dix, par l'intensification de ces oppositions nationales

Si l'arrivée de Jacques Delors à la présidence de la Commission en 1985 avait coïncidé avec la réinscription, sur l'agenda européen, de certains sujets sociaux, cette " relance » a en effet été de courte durée et les projets peinent à se traduire concrètement. Dans un contexte général de récession et de mise en place de politiques dites d'austérité dans les pays de l'UE, les services de la DG V ont fait, surtout, l'expérience de constantes réévaluations, redéfinitions, voire remises en question, par certains gouvernements, des compétences de la Commission dans le domaine

12 P. Bezès, « Les hauts fonctionnaires croient-ils à leurs mythes? L'apport des approches cognitives à l'analyse des engagements dans les politiques de réforme de l'Etat. Quelques exemples français (1988-1997) », Revue Française de Science Politique, 50 (2), 2000.

13 Voir aussi sur ce point: M. Abélès, I. Bellier, «La Commission européenne du compromis culturel à la culture politique du compromis », Revue Française de Science Politique, 46 (3), 1996.

14 A l'image du témoignage de l'ancien commissaire français, Robert Marjolin : « Le fait est que, de manière inévitable, les membres de la Commission, aussi attachés qu'ils soient à l'idée européenne, ont eu à prendre en considération les positions des gouvernements nationaux, au risque sinon de renoncer à toute efficacité. Un aspect essentiel de leur travail a consisté à tenter de réconcilier ce que leur dictait l'intérêt général avec ce qu'ils connaissaient des ambitions et des objectifs des différents gouvernements, y compris le gouvernement du pays auquel ils appartenaient. »(R. Marjolin (1989), Memoirs 1911-1986 : architect of European unity, London, Weidenfeld and Nicolson, 1998, cité par G. Edwards et D. Spence (dir.), The European Commission, London, Longman, 1994, (p.6). Sur les usages de cette littérature : D. Georgakakis, « Les réalités d'un mythe : figure de l'eurocrate et institutionnalisation de l'Europe politique» in Dubois Vincent, Dulong Delphine (dir.), La question technocratique. De l'invention d'une figure aux transformations de l'action publique, Strasbourg, Presses Universitaires de Strasbourg, 1999. 
social : outre l'opt out du gouvernement anglais concernant le chapitre social de Maastricht ${ }^{15}$, les veto posés à l'adoption de programmes sociaux au Conseil et les procédures de contestation devant la Cour de justice se multiplient, notamment à l'initiative du gouvernement allemand, jusque-là l'un des plus constants soutiens de la DG V. Ces expériences alimentent chez ses agents un sentiment de précarité, dont les formes sont autant symboliques que matérielles.

"Ici, on fait quelque chose où il n'y a pas de consensus réel, de consensus politique, nous avons des problèmes sans cesse. Par exemple, ici, dans notre unité, on a des programmes d'action en faveur des personnes âgées et aussi pour lutter contre l'exclusion sociale. Et bien, le Royaume Uni a saisi la Cour [de justice] européenne et a posé la question de savoir :'Est-ce qu'il est légal, est-ce qu'il est conforme au Traité que la DG V dépense de l'argent, provenant du budget de la Communauté sur la base de ces lignes ?' Alors la Cour européenne a dit : 'Bon, il faut vérifier ça, mais pour le moment, il vaut mieux ne pas dépenser cet argent'. C'est-à-dire que du coup, deux tiers de l'unité sont bloqués, parce qu'effectivement ces programmes-là exigent de faire des dépenses, donner de l'argent à des acteurs, à des ONG. » (Membre de la DG V, direction «sécurité sociale et programmes d'action sociale », novembre 1996, Bruxelles)

L'évocation de ce contexte nourrit conjointement une interprétation de l'avenir des politiques sociales européennes qui met l'accent sur les résistances nationales à toute initiative dans ce domaine, et insiste, dans un même mouvement, sur le nécessité de concevoir le travail des services de la Commission comme une contribution résiduelle.

«Non, les paramètres ne sont pas formidables, même si actuellement, là, la conjonction des paramètres du premier semestre de cette année donne un petit peu de positif avec des changements politiques au Royaume-Uni et en France, une CIG [conférence intergouvernementale] qui devrait un petit peu renforcer la politique sociale, une affaire Renault ${ }^{16}$ dont on n'est pas responsable, mais enfin ça oblige les décideurs politiques à réfléchir à l'impact réel des directives. Moi je fais le pronostic, qu'à l'Ouest comme à l'Est, ce n'est pas par le sommet que tous les changements se feront, c'est souvent par la base, ou par les accidents de parcours. Mais sur le point de savoir si au niveau politique l'Union européenne est capable de porter un message global qui comprenne le social suffisamment, je ne suis pas très très optimiste, plutôt réaliste. » (Membre de la DG V, direction «dialogue social », juin 1997, Bruxelles)

15 L'opt out correspond à la revendication par un Etat membre d'une mesure dérogatoire lui permettant de ne pas s'associer à l'un ou l'autre des projets et dispositifs adoptés dans le cadre communautaire. L'opt out britannique évoqué ici est l'un de ceux émis par le gouvernement de John Major au moment du Traité de Maastricht, aboutissant à la signature à onze du " protocole social » et à sa simple annexion au Traité. Ce texte, qui visait à relancer l'activité politique et réglementaire en matière sociale sans toutefois constituer un engagement contraignant, a été réintégré dans le Traité d'Amsterdam suite à l'arrivée au pouvoir de Tony Blair en 1997 et à la levée consécutive du veto anglais. D'autres clauses d'opt out ont été négociées par le gouvernement Major dans cette même période, autorisant notamment les employeurs britanniques à contourner la réglementation européenne en matière de temps de travail par des accords spécifiques avec leurs salariés.

16 En évoquant l'affaire Renault, la personne interrogée fait ici référence au conflit lié à l'annonce, en février 1997, de la fermeture d'une usine du groupe située à Vilvorde et à ses répercussions : celle-ci est en effet l'occasion d'une mobilisation syndicale revendiquant une dimension européenne à l'action des salariés (manifestations et communiqué communs des syndicats français et belges dès les premiers jours) et aux réponses politiques qu'elles appellent. Directement interpellée, la Commission répond dans un premier temps, par l'intermédiaire de Karel Van Miert, commissaire belge à la Concurrence, qui annoncera la suppression de l'aide financière au site de Renault à Valladolid, mais incite surtout les syndicats à porter plainte pour non respect des procédures européennes d'information des travailleurs. Cette plainte donne lieu à un jugement favorable aux plaignants (Cour d'appel de Versailles, 7 mai 1999), qui inspire une seconde directive européenne sur l'information et la consultation des salariés adoptée en 2002 (directive 2002/14/CE du 11 mars 2002). Voir sur ce point : C. Didry, « Le comité d'entreprise européen devant la justice. Mobilisation du droit et travail juridique communautaire », Droit et société, 41, 1999. 
C'est à la lumière de cette mémoire propre au service, constituée par la sédimentation des conflits et des échecs vécus dans un passé plus ou moins proche, que ces agents interprètent le silence maintenu par les instances intergouvernementales sur le sujets sociaux, durant toute la période précédant l'ouverture des négociations d'adhésion ${ }^{17}$. Le désintérêt de la plupart des Etats membres pour la dimension sociale de l'élargissement, mais aussi et surtout le caractère potentiellement conflictuel du dossier, semblent acquérir aux yeux des fonctionnaires interrogés une réalité incontestable. Celle-ci vient alors justifier avec la même force de l'évidence, le renoncement à défendre une conception extensive de l'acquis social. En rationalisant de la sorte cet ajustement à la baisse de leurs ambitions institutionnelles, ils laissent ainsi entendre que les seules frontières légitimes du possible et du dicible en matière sociale sont celles qui sont établies par les instances intergouvernementales. Comme l'illustre l'extrait d'entretien suivant, conduit avec un agent qui quittera d'ailleurs le service pour rejoindre ensuite la haute administration française, l'acquis social communautaire à revendiquer face aux pays candidats devient ainsi, par une sorte de glissement implicite, moins la synthèse d'un modèle social européen que ce que l'ensemble des gouvernements en place dans les Etats membres sont prêts à en reconnaître.

«Je pense qu'il faut d'abord qu'on attende le nouveau Traité. Personnellement c'est ma ligne. On a beaucoup parlé, A [commanditaire du rapport] a beaucoup parlé, de l'acquis implicite, mais je ne crois pas qu'il y ait d'acquis implicite, je ne suis pas d'accord avec elle là-dessus, ou alors il faudrait vraiment que tous les gouvernements changent dans la Communauté. Si tous les gouvernements changeaient dans la Communauté, si tous les gouvernements devenaient socialistes, on pourrait dire: 'voilà, il y a un acquis implicite'. Et tout le monde le reconnaîtrait. Personnellement je crois que ce n'est pas vrai, je crois qu'on n'arrivera pas à obtenir l'accord des Quinze - parce que c'est bien ça qu'il faut obtenir-, sur l'idée qu'il y a un acquis implicite (...). Je ne pense pas que ce soit possible de défendre l'idée d'un certain niveau de protection sociale (...). Je ne crois pas du tout qu'à un moment, on pourra mettre dans les négociations cette idée d'un niveau minimal de protection sociale comme condition pour rentrer. » (Membre de la DG V, direction « sécurité sociale et programmes d'action sociale », juin 1997, Bruxelles)

C'est bien la volonté de se conformer à une certaine conception du rôle de l'administration communautaire qui est ici soulignée dans ces argumentaires. Les justifications de l'adoption d'une définition juridique, comme du refus d'endosser un discours explicitement politique sur la dimension sociale de l'élargissement, constituent les deux facettes d'une même représentation de la Commission et de ses membres comme des agents neutres, au service de principes définis en dehors d'eux. A travers le choix du droit, ils prennent l'apparence de simples porte-parole, affichant ainsi leur soumission à l'autorité des principes juridiques et effaçant la dimension créatrice contenue dans le travail de sélection des textes et de leur interprétation ${ }^{18}$. C'est d'autre part au nom de cette même " neutralité » de l'administration que le rejet d'une définition extensive de l'acquis social européen est ensuite justifié au motif que sa défense reviendrait à revendiquer une autorité que ni les Traités, ni l'élection ne conféreraient à l'institution.

\footnotetext{
17 Par ailleurs, pendant les négociations proprement dites, aucun débat politique communautaire n'a été ouvert à notre connaissance sur cette question. Voir pour une analyse de ce « silence politique » : C. Robert, « Les acteurs communautaires face à la dimension sociale de l'élargissement de l'Union aux pays d'Europe centrale et orientale (1989-1998) : l'impossible émergence d'un débat », in H. Oberdorff et G. Guillermin (dir.), La cohésion économique et sociale : une finalité de l'Union européenne, Paris, La Documentation Française, 2000.

$18 \mathrm{P}$. Bourdieu, « La force du droit. Eléments pour une sociologie du champ juridique », Actes de la Recherche en Sciences Sociales, 64, 1986, (p.6)
} 
"C'est un peu la tradition administrative au sein de la Commission, que nous soyons très centrés sur les normes juridiques. On ne peut pas dire : 'il faut que tu fasses ça ou ça', s'il n'y a pas de base juridique, parce que ça impliquerait que soudainement, la Commission donne des ordres aux pays tiers, dans un domaine où elle ne peut rien dire au sein de l'Union [...]. Alors, ce ne serait pas très logique, n'est-ce pas? Alors nous avons le Traité, et toutes les normes secondaires, ce sont un peu les règles du jeu, il faut les accepter, comme elles existent aujourd'hui. Oui ou non, si oui, on devient Etat membre. (...) Et une partie de mon boulot, c'est que je ne peux pas dire: 'ça c'est bon, ça c'est mauvais', 'toi tu es le bon élève, et toi tu es le mauvais'. Je ne peux pas le dire. Même si j'avais la conviction personnelle qu'il faut faire ça, je ne pourrais pas. Et si je le laisse faire, j'aurais un problème politique parce que dans ce cas, on dirait 'mais non la Commission ne peut pas faire ça'. » (Membre de la DG V, direction « sécurité sociale et programmes d'action sociale », novembre 1996, Bruxelles)

Soulignant constamment les contraintes de légitimité qui s'imposent à eux, en tant que membres de la Commission, et reproduisant à ce titre de manière presque idéal-typique le discours que l'institution construit sur elle-même et son a-politisme ${ }^{19}$, les arguments déployés laissent ainsi entrevoir qu'un des enjeux de cette controverse réside notamment, pour ces agents, dans leur capacité à incarner leur rôle institutionnel, à attester qu'ils s'y conforment et y adhèrent. C'est ce qu'évoque par exemple cet éloge du pragmatisme auquel se livre un des agents interrogés, qui occupe à la $\mathrm{DG} \mathrm{V}$ son premier poste : présentée comme une nécessité quasi-psychologique (dictée par la prise en compte du pouvoir de fait limité de l'administration communautaire), érigée en impératif « moral » (lié au respect de principes démocratiques), la "capacité d'adaptation » au contexte politique est ici également construite, de manière significative, comme une qualité professionnelle indispensable «au bon fonctionnaire ».

«Tout ça, c'est seulement une appréciation pragmatique de l'impact que je peux avoir. (...) Je crois que [en tant que fonctionnaire] si on est idéaliste, si on veut créer l'Europe parfaite, l'harmonie totale, on est très frustré parce que c'est une administration publique. Je crois que si on veut survivre mentalement ou être content, il faut être un bon fonctionnaire, peut-être en français on dirait un bon technocrate, quelque chose comme ça, quelqu'un qui fait bien son boulot, qui montre un sens des responsabilités. Je ne veux pas dire quelqu'un qui est indifférent vis-à-vis de l'Europe, mais si on espérait toujours qu'on peut sauver le Monde et l'Europe avec chaque activité, ce serait très frustrant, je crois bien. Parce que si on a quinze Etats membres, pratiquement tout ce qu'on fait est une espèce de compromis. Il faut toujours être prudent, au moins ici. Il faut respecter que nous sommes différents. Et si vous avez une opinion claire, il faut toujours être diplomatique, et parfois, il y a une certaine contradiction entre diplomatie et efficacité technique. » (Membre de la DG V, direction "sécurité sociale et programmes d'action sociale », juin 1997, Bruxelles.)

L'histoire de l'administration communautaire abonde en exemples de services qui, plus que ceux de l'emploi et des affaires sociales, n'ont pas hésité à revendiquer et exercer plus fermement leur pouvoir d'initiative et à exiger d'un Conseil réticent une extension de leurs prérogatives ${ }^{20}$. Ces stratégies sectorielles différenciées renvoient notamment au fait que la

19 Sur ce « rapport au politique » des eurofonctionnaires, voir : C. Robert , « Doing politics and pretending not to. The Commission's role in distributing aid to Eastern Europe », in Smith Andy (ed.), Politics and the European Commission. Actors, interdependence, legitimacy, London, Routledge, 2004.

20 C'est particulièrement le cas de directions générales en charge de sujets très tôt construits comme communautaires (marché intérieur, concurrence, dans une certaine mesure politique agricole), mais ces stratégies peuvent être également le fait de services plus récents et bien moins établis, comme l'a montré Michelle Cini à propos de la direction générale en charge de l'environnement dans les années 1990. M. Cini, « La Commission européenne, lieu d'émergence de cultures administratives. L'exemple de la DG IV et de la DG XI », Revue Française de Science Politique, 46(3), 1996. 
conception forgée par les fonctionnaires de leur rôle n'est pas une donnée intangible ; pas plus qu'elle n'est strictement identique en tout lieu de l'institution. Comme le laisse entendre cette responsable des programmes à destination des pays candidats, membre d'une autre direction générale, c'est une interprétation particulièrement rigoureuse de ces prescriptions de rôle que donnent à voir les agents de la DG.

«C'est assez curieux, parce que très souvent, on est face à des gens qui, à titre personnel, ont une grande sensibilité sociale, et qui, dans le contexte de leur travail-est-ce que c'est pour se conformer au moule et puis pouvoir avoir une promotion après, j'en sais rien finalement défendent des positions qui ne leur ressemblent pas à titre privé. Il y a une dichotomie. (...) Parce qu'ils sont pris dans la logique institutionnelle et dans un cadre donné. C'est très curieux, moi je trouve ça très choquant, parce que c'est comme s'ils cessaient d'être citoyens à partir du moment où ils sont fonctionnaires à une position suffisamment élevée. Bien sûr qu'à l'extérieur on doit avoir le discours de la Commission, même si on ne l'approuve pas, mais quand on est dans les discussions où on peut influer, il est curieux qu'il y ait une telle dichotomie. Je suis assez frappée de voir que des gens qui ont une approche très sociale préfèrent l'évacuer, parce qu'ils se disent que sinon ça ne passera pas, et que, peut-être, ça n'en vaut pas la peine. " (Membre de la DG IA, service «Phare ${ }^{21}$, juin 1997, Bruxelles)

Les postures adoptées par ces agents sont le produit d'un ensemble de déterminants que les données recueillies dans le cadre de cette enquête ne permettent pas d'explorer systématiquement. Elles reflètent pour partie la prudence de fonctionnaires en début de carrière ou en demande de mobilité, particulièrement nombreux dans ces services, et évitant autant que possible d'endosser des positions controversées susceptibles de peser lourdement sur leurs carrières 22 . Elles traduisent aussi plus généralement les efforts déployés par ces personnels pour conjurer leur marginalité en attestant de leur bonne volonté professionnelle et de la modestie de leurs ambitions institutionnelles. L'hyperconformisme dont ils témoignent dans le cadre du débat sur l'élargissement et qui les conduit à se retrancher derrière le droit plutôt que d'endosser une position explicitement politique (et potentiellement conflictuelle) peut ainsi se comprendre comme un des produits de la situation de domination dans laquelle ils se trouvent placés au sein de l'administration communautaire.

\section{Comment parler du social sans risque ? Contraintes réthoriques et auto-censure}

Limitant leur capacité à jouer avec certaines des règles qui gouvernent le fonctionnement de l'administration européenne, les positions de ces agents dans l'espace institutionnel européen les conduit également à intérioriser d'autres formes de contraintes qui sont autant d'obstacles à la formalisation d'un discours en faveur des enjeux sociaux et à sa réception au sein de la Commission. C'est notamment ce qu'évoque leur refus de mobiliser certains arguments, proposés dans le rapport sur la dimension sociale de l'élargissement, au motif qu'ils pourraient contribuer à affaiblir encore leur crédit et alimenter les critiques de leurs détracteurs. A l'instar des agents d'Etats en charge de la culture, étudiés par Vincent

\footnotetext{
${ }^{21}$ Le service Phare était en charge de la gestion du programme du même nom, destiné à apporter une assistance technique à la « Transition », puis à « la préparation à l'adhésion » aux pays d'Europe centrale et orientale.

22 La défense d'une position ou d'un dossier suscitant un vote négatif au collège ou même encore de la part des représentants des Etats membres peut avoir, comme le note Mark Pollack, de durables répercussions sur les carrières de ceux qui ont porté le projet. M. A. Pollack, «Delegation, agency and agenda setting in the European Community », International Organization, 51 (1), 1997.
} 
Dubois $^{23}$, les membres de la DG V se placent ici essentiellement dans une position défensive, et les registres de justification qu'ils déploient paraissent moins choisis qu'imposés, dictés par les remises en cause dont l'existence même d'une politique sociale européenne fait constamment l'objet. S'apparentant ainsi à une forme d'autocensure, ces pratiques permettent plus généralement de souligner l'inadéquation des ressources de la DG V avec la rhétorique et les modes de sélection des problèmes qui prévalent au sein de la Commission.

Les débats témoignent d'abord des contraintes, voire des handicaps que représente pour ces services la montée en puissance, dans la bureaucratie communautaire, de l'expertise économique. Ils portent en effet sur le projet de préparer une «étude d'impact 》 destinée à recenser les conséquences possibles, sur le plan social, de l'intégration des pays d'Europe Centrale et Orientale dans l'Union. Initiée par les promoteurs du document sur le modèle social européen, l'idée d'une telle étude vise, comme leur rapport, à attirer l'attention sur des enjeux qu'ils estiment insuffisamment pris en compte.

La nature et la forme choisies pour cet exercice illustrent bien les conditions requises pour qu'un enjeu puisse conquérir une visibilité au sein de la Commission. Travail de prospective économique, offrant un contenu mesurable, éventuellement chiffré, il est un instrument classique de la mise en alerte dans cet univers administratif. En outre, dans le contexte de l'élargissement, il constitue, avec l'examen des conditions de transposition du droit communautaire dans les pays candidats, l'un des éléments clefs du cadrage administratif des négociations d'adhésion. La réflexion conduite sur les modalités de l'intégration des $\mathrm{PECO}$, et les réformes communautaires qu'elle implique, fait en effet une place importante à la prospective budgétaire et financière dans la mesure où elle s'inscrit dans un contexte de renégociation, au sein de l'Union, du budget et des principales politiques européennes (politique agricole commune et fonds structurels). Les études d'impact qui sont préparées dans les services sont donc mobilisées à la fois pour identifier d'éventuelles exigences opposables aux futurs entrants, et pour repérer les réformes des dispositifs communautaires qui seraient rendues nécessaires par leur adhésion.

"C'était à l'époque où on préparait l'étude d'impact pour l'Agenda 2000, et il n'y avait rien de prévu pour le secteur social. Donc j'ai dit à X [directeur général adjoint de la DG IA] : 'ça ne peut pas aller, il faut faire quelque chose'. Il m'a répondu: 'Allez-y, faites vos termes de référence.' [document contractuel indiquant aux experts les points devant être abordés dans le rapport qui leur est confié] Donc comme j'avais eu son accord, j'avais préparé des termes de référence, sur des questions comme : Qu'est-ce que ça ferait l'élargissement? Pour les pays de l'Est qu'est-ce que ça allait impliquer pour eux? Quels étaient les efforts qu'ils allaient avoir à faire? Et bon, combien ça allait leur coûter en termes de temps de travail, de structures à mettre en place, financièrement, etc. Et la même chose pour les pays de l'Ouest. Ces termes de référence ont été ensuite approuvés par la petite équipe élargissement, qui était auprès de $X$. Mais il a dit: 'On ne va pas faire une étude d'impact sans l'accord de la DG V.' Or, la DG V était contre. (...) Le résultat c'est que quand on n'a pas d'étude d'impact, un, les pays de l'Est pensent que si on ne mentionne rien, c'est pas important, et deux, nous, de nôtre côté, on ne va pas développer d'instruments d'aide. Par exemple, on a fait une étude d'impact dans le domaine agricole, on va décider de mettre dès avant l'adhésion de ces pays, dès qu'on aura ouvert les négociations, des instruments qui leur permettront de se préparer dans le domaine agricole. Dans le domaine social, on ne le fera pas, puisqu'on n'a pas fait d'étude d'impact." (Commanditaire du rapport, membre de la DG IA, service « Phare », août 1998, Avignon)

23 V. Dubois, «L'art et l'Etat au début de la IIIème République, ou les conditions d'impossibilité de la mise en forme d'une politique », Genèses, 23, 1996. 
Le refus catégorique opposé par la DG V à ce projet met en évidence les obstacles que soulève pour ses agents le fait de penser dans le langage et les catégories d'analyse perçus pourtant comme dominants au sein de la Commission. Leur difficulté à s'approprier un tel registre, qui n'est peut-être pas étrangère à la présence, proportionnellement faible, des économistes de formation au sein de la direction générale, se traduit d'abord par des commentaires soulignant l'inadéquation des grilles de lectures économiques aux problématiques spécifiques du secteur social. Ainsi, pour cet agent en charge de programmes communautaires destinés à soutenir les syndicats est-européens, ancien inspecteur du travail français devenu fonctionnaire européen, une analyse en termes économiques du dialogue social rendrait nécessairement mal compte de son intérêt et conduirait à en minorer les enjeux.

«Moi, je suis carrément très très sceptique sur cette notion d'étude d'impact, je sais que c'est normal, je pense que si on était Premier ministre, ce serait la question qu'on poserait à nos collaborateurs, mais la politique sociale c'est très difficile à juger et quand on est dans la zone la plus qualitative qui est le dialogue social, qu'est-ce que je vais mesurer moi comme impact? Moi, quand on m'a posé la question, j'ai répondu : 'Mais l'impact, non seulement ça ne coûte rien un bon dialogue social, mais en plus on a tendance à y gagner. 'Moi, je n'ai que l'affaire Renault, sous la main, à montrer. Ne pas consacrer suffisamment d'attention au dialogue social, c'est risquer des affaires Renault, donc un gâchis économique et un gâchis social. (...) Et puis, c'est tout le malheur de la construction européenne, c'est-à-dire qu'on est basé sur une ligne principalement économique et financière. Et si on fait passer tout ça à la moulinette de l'économique et du financier, on est perdu, parce que l'impact du social ne fait pas le poids par rapport à l'impact de la politique agricole, qu'on peut déjà sans doute un peu mieux mesurer. » (Membre de la DG V, direction « dialogue social », juin 1997, Bruxelles)

Le malaise qu'exprime le personnel à l'idée de mobiliser ce registre peut ensuite être rapporté aux usages qui en ont été faits, pendant la dernière décennie, dans les luttes politiques et administratives sur les sujets sociaux. C'est en effet au nom d'une expertise économique que certaines directions générales concurrentes, aux positions et à la légitimité mieux établies au sein de la Commission, ont tenté d'imposer leurs propres conceptions des politiques sociales. Conduites notamment par les services en charge du marché intérieur (DG $\mathrm{XV}$ ) et de l'économie (DG II), ces entreprises visant à contester le monopole des acteurs nominalement en charge du social sur leur territoire ont d'autant plus de poids qu'elles reproduisent et s'appuient sur les stratégies engagées par les grands organismes financiers internationaux.

La question des retraites offre une illustration exemplaire de ces stratégies. Les directions générales en charge du marché intérieur et des affaires économiques et financières concentrent en effet leur activité sur ces sujets, au nom notamment au de leurs prérogatives en matière de liberté de circulation des capitaux (liée au marché unique). Leurs documents font ainsi une large place aux fonds de pension par capitalisation. Ils insistent par ailleurs sur la nécessité de préserver les grands équilibres macro-économiques dans la perspective de l'adhésion, faisant apparaître plus ou moins explicitement les systèmes de retraites par répartition comme les principaux responsables des déficits budgétaires des pays candidats ${ }^{24}$. Dans le contexte de

\footnotetext{
24 «Par exemple, la DGXV (marché intérieur) a publié ce Livre Vert sur les fonds de pension, qui posent des problèmes de mouvements des capitaux, de fiscalité, etc. Alors qu'en Europe, on garde quand même un socle par répartition important. Là où ça ne va pas en Europe de l'Est, c'est que les pays de l'Est auraient souhaité carrément éliminer le système par répartition, et dans la DG II (affaires économiques) et la DG XV, il y avait pas mal de gens qui étaient d'accord et qui encourageaient cette approche. (...) Quand on discute avec eux, ils disent : 'Pourquoi vous ne voulez pas aider les PECO à mettre en place des systèmes par capitalisation? Nos systèmes à nous survivent de plus en plus difficilement, coûtent une fortune'». (Commanditaire du rapport, membre de la DG IA, « service Phare », août 1998, Avignon)
} 
l'élargissement, ces stratégies trouvent en outre des liens très directs avec le travail d'expertise conduit par les organismes financiers internationaux sur le terrain est-européen. Les discussions engagées avec les pays candidats par ces directions générales s'inspirent ainsi explicitement des conclusions du rapport de la Banque Mondiale « prévenir la crise des retraites ${ }^{25}$, qui a été très largement diffusé auprès des gouvernements est-européens de la Transition.

Ces lectures dominantes des politiques sociales qui visent principalement à en souligner les coûts éclairent les réticences des services de la DG V à l'égard des instrumentalisations possibles d'une lecture économique. Elles s'inscrivent en outre dans un contexte européen et international où se multiplient les discours dénonçant la crise financière des Etats providence. Vécu comme imposé de l'extérieur, le langage de l'économie est perçu comme véhiculant nécessairement, et quel qu'en soit l'interprète, une hiérarchisation implicite des secteurs d'action publique défavorable aux politiques sociales. Ce rapport à l'expertise économique est également emblématique de la position défensive adoptée par ces services : les instruments et stratégies qu'ils déploient sont essentiellement construits en réponse aux attaques et critiques dont ils font l'objet. Tandis que l'expertise économique est principalement appréhendée à travers les usages qu'en font leurs adversaires au sein et autour de la Commission, ils choisissent en outre de renoncer à cette ressource argumentative dont ils reconnaissent pourtant l'efficacité symbolique.

«Et $Y$ [fonctionnaire de la DG $V$, chargée officiellement des relations avec la DG IA] furieuse répond en disant : 'Notre politique a toujours été de refuser des études d'impact sur les coûts liés au secteur social parce que le social c'est un investissement. On ne peut pas évaluer ce que ça rapporte en tant qu'investissement, par contre on évalue toujours ce que ça coûte, y en a marre. On va encore avoir tout le monde contre nous en disant que ça coûte cher, on est contre le principe de faire une étude d'impact. " (Commanditaire du rapport, membre de la DG IA, service « Phare », août 1998, Avignon)

Si la seconde controverse opposant les agents de la DG V aux auteurs du rapport aboutit pour sa part à asseoir le monopole du droit comme seul registre légitime d'énonciation du social, elle procède néanmoins d'une même logique défensive. En refusant de sortir d'un discours juridique sur l'acquis communautaire, et d'accorder, comme les y invite le rapport, une légitimité équivalente à des principes qui ne sont pas inscrits dans le droit européen, la plupart de ces fonctionnaires adoptent ainsi une position de repli. Privilégiant la défense du droit existant, considéré comme menacé, à la valorisation d'autres formes d'action publique pourtant initiées au sein de ces services, cette décision ne fait pas, par ailleurs, l'unanimité. Ces controverses sur les formes légitimes d'énonciation du social montrent ainsi comment la valorisation des ressources juridiques dans le cadre de l'élargissement réactivent les concurrences au sein même des services et en modifient les rapports de force internes.

La défense d'une conception élargie de l'acquis communautaire en matière sociale, telle qu'elle est mise en œuvre dans le rapport, se fonde en effet sur une critique du droit comme instrument de description de la réalité sociale européenne, qu'une partie du personnel de la DG V va systématiquement contester. Prenant la forme de corrections écrites sur le rapport, leurs commentaires semblent notamment viser à éviter toute formulation portant atteinte, même de manière purement rhétorique, aux directives et règlements existants.

25 World Bank, Adverting the old age crisis. Policies to protect the old and promote growth, World Bank Policy Research Report, Washington D.C., 1994. La DG II a ainsi organisé en 1996 deux rencontres avec les pays candidats et des représentants de la Banque Mondiale pour évoquer le rapport. 
Parmi les arguments contestés par les agents de la DG V, figure d'abord la «faiblesse » de la réglementation existante : les auteurs du rapport justifient en effet leur défense d'une définition extra-juridique du modèle social européen, au motif que les domaines concernés par le droit sont très limités et les normes communautaires peu exigeantes. Ces propos suscitent chez les eurofonctionnaires des réactions offusquées et les modifications qu'ils apportent montrent clairement qu'il n'est pas question pour eux de faire apparaître les directives comme un droit résiduel et peu significatif, et encore moins d'en minorer l'importance.

C'est ce qu'évoque ce courrier émanant de la direction en charge des directives sur la santé et la sécurité au travail exigeant la suppression de la formule suivante - "la directive est moins exigeante que la majorité des règles nationales»- et concluant "La DG IA aurait dû se renseigner auprès des services compétents avant de rédiger une affirmation que nous savons bien contraire à la réalité. » (Lettre d'un membre de la DG V, en date de décembre 1996)

Un second argument, récusé lui aussi, consiste à établir qu'une définition extensive de l'acquis social - comprenant notamment un ensemble de principes relatifs à la sécurité sociale et à la protection sociale - peut être attestée par la relecture de grands " textes fondateurs »-Traité de Rome, Acte Unique, Traité de Maastricht - et de l'ensemble des documents - de statuts divers qui ont jalonné les développements de la politique sociale communautaire. Mais cette opération d'assimilation - qui consiste à faire rentrer dans l'acquis des principes auxquels ne correspond pas de formulation juridique dans le droit communautaire - aboutit indirectement à considérer que des textes non juridiquement contraignants (comme les Recommandations) ont la même valeur et la même légitimité que des directives européennes. Cette hybridation entre différents ordres normatifs est rapidement dénoncée par certains fonctionnaires, comme l'illustre cette correction transmise aux consultants :

"Par ailleurs, je vous fais remarquer que la Charte communautaire des droits sociaux fondamentaux n'est pas un acte juridique, mais une déclaration des Chefs d'Etats et de Gouvernements, donc de ce fait, elle ne peut pas être un texte de base équivalent aux textes des Traités. » (Lettre d'un membre de la DG V en date de juillet 1997)

Ici encore, la posture défensive adoptée par les services de la DG V n'est pas sans lien avec le sentiment largement partagé d'un risque de marginalisation des enjeux sociaux dans le contexte de l'élargissement. Les années précédant l'ouverture des négociations d'adhésion ont en effet été le théâtre d'une série d'événements qui les conduisent à considérer que même le droit existant dans ce domaine pourrait être remis en cause.

Ce sont d'abord les déclarations de certains hommes politiques, à l'Est, mais aussi au sein de l'Union, qui évoquent la nécessité, dans la perspective de l'élargissement, de passer outre l'acquis existant : le Premier ministre britannique, le conservateur John Major, mène ainsi une véritable campagne, à l'occasion de ses déplacements en Europe de l'Est, contre l'acquis social communautaire, rappelant à qui veut l'entendre que rien n'interdit aux gouvernements des pays candidats d'exiger, comme les Britanniques, un opt out en matière sociale, dans le cadre de leur intégration dans l'Union ${ }^{26}$. Au sein même de l'administration européenne, certaines initiatives contribueront à confirmer les eurofonctionnaires en charge des politiques sociales dans le sentiment que les différents secteurs d'intervention communautaire ne bénéficient pas d'une égale considération : ainsi, dans une première version du document recensant le droit

\footnotetext{
26 Une dépêche de l'agence Reuters indique que John Major aurait notamment tenu les propos suivants au gouvernement tchèque en octobre 1996 : "On vous a dit à vous, les Tchèques, que le chapitre social de Maastricht était inclus dans l'acquis communautaire et que vous n'aviez pas le choix de le reprendre ou pas. De notre point de vue, cette obligation n'existe pas. » (Reuter Newswire, 14 octobre 1996). Voir sur les discours du gouvernement britannique concernant l'acquis social communautaire : K. E. Jorgensen, « The social construction of the acquis communautaire : a cornerstone of the European edifice ", European Integration online Papers (http://eiop.or.at/eiop/texte), 3(5), 1999.
} 
communautaire devant être repris dans les législations des pays candidats $^{27}$, seules les directives et règlements relatifs au social ne sont pas mentionnées. Présentée ensuite comme une " erreur » par la direction générale en charge du marché intérieur, "l'affaire » aurait néanmoins nécessité l'intervention du Conseil puis du Collège pour être « réglée $»^{28}$

Construit dans l'adversité, le rapport privilégié qu'entretiennent les services de la DG V au droit est néanmoins inégalement partagé par ses différents agents. De manière significative, les oppositions les plus vives aux jeux avec le droit qu'implique la définition d'un modèle social européen proviennent des unités qui peuvent faire des directives et règlements existants une ressource pour l'action. A l'inverse, les services qui ne disposent pas de tels instruments, et qui s'efforcent de promouvoir leurs propres objets par l'intermédiaire de textes ou de pratiques non juridiquement contraignants ${ }^{29}$ se sont montrés beaucoup plus favorables à cette initiative. Comme le rappelle notamment l'un des membres de ces services, en charge du dialogue social :

«A mon niveau, c'est clair que j'ai toujours défendu l'idée que l'acquis social n'a de sens que si on le voit de manière large et si on y intègre effectivement l'emploi, le dialogue social, la protection sociale, donc des éléments qui ne sont pas de la législation en tant que telle. C'est tout de même le discours qui est tenu majoritairement à la DG V. même si on n'en a jamais vraiment pris les moyens, donc on n'avait rien contre cette approche. Mais c'est vrai que le travail des consultants a certainement été compliqué par des points de vue divers et bon, certains collègues qui ne travaillaient que sur des matières très juridiques ne pouvaient que défendre un petit peu l'inverse de ce que je dis, en pensant que leur propre matière était au centre, et donc la plus importante, et donc du coup, il y a eu des hésitations dans la ligne de conduite générale. » (Membre de la DG V, direction "dialogue social», septembre 1998, Bruxelles)

Les débats initiés autour de la définition de l'acquis communautaire font ainsi écho aux concurrences qui divisent la direction générale et contribuent peut-être même à les exacerber. La position de repli adoptée par la DG V consiste en effet à passer sous silence l'activité d'une partie de ses services pour préserver ce qui est redéfini comme le cœur de sa mission : à l'affirmation d'une hiérarchie, d'une supériorité des conquêtes réglementaires sur d'autres réalisations s'ajoutent les « sacrifices » qu'il faut consentir pour préserver l'acquis juridique. A travers ces remarques acerbes sur le pointillisme des unités juridiques, c'est tout à la fois le rôle joué par le droit dans la construction des rapports de force au sein des services et plus largement de la Commission, et l'imposition, à la faveur de l'élargissement, d'une certaine

\footnotetext{
27 Il s'agit plus précisément du Livre Blanc sur le marché intérieur, dont la législation constitue une partie très importante du droit communautaire. La majorité des directives en matière sociale sont liées juridiquement au fonctionnement du marché intérieur : l'égalité des chances pour les femmes et les hommes, la coordination des régimes de sécurité sociale, la santé et la sécurité au travail, les droits et conditions de travail, les produits de tabac (teneur en goudron et étiquetage). Livre Blanc «préparation des Etats associés de l'Europe centrale et orientale à leur intégration dans le Marché Intérieur de l'Union », 1995, [COM(95) 163 final].

28 «En fait, la DG XV avait laissé complètement de côté tout l'acquis communautaire en matière sociale, il était purement et simplement oublié, dans une première version qu'on avait eue. Alors, après (...) effectivement, il y a eu une intervention française dans un Conseil "Affaires générales » où on avait la Présidence, et où le ministre des Affaires étrangères, Juppé à l'époque, avait demandé à la Commission de refaire une version du Livre Blanc qui intégrerait les points qui avaient été mentionnés, dont effectivement le point social. » (Membre du cabinet de Martine Aubry, alors ministre de l'Emploi et des Affaires sociales, avril 1997, Paris)

29 Comme les recommandations, les opinions, et autres publications de la Commission ou encore dans un registre complémentaire la mise en place de groupes de concertation ou de travail, la création de réseaux d'experts.
} 
lecture de l'histoire et des ambitions de la direction générale en matière sociale qui sont ici soulignés.

\section{L'intériorisation de l'illégitimité du social}

La conscience aiguë, partagée par les agents de la Commission, de la fragilité de l'édifice social européen, et de la précarité de leur situation sur les plans institutionnel et politique, oriente ainsi leur perception des enjeux liés à l'élargissement. Si elle détermine les " coups » qu'ils s'autorisent, et restreint le choix des arguments qu'ils se permettent de déployer sur la scène bruxelloise, elle informe aussi, plus profondément, la manière dont ils perçoivent leurs objectifs et raison d'être au sein de l'Union. Tout autant que des considérations tactiques, les positions qu'ils adoptent reflètent une forme d'intériorisation de l'illégitimité de leurs pratiques et de leurs référents pour l'action.

Cette « crise de légitimité » prend d'abord la forme d'une prise de distance avec les ambitions originelles du service. Elle se traduit notamment par le refus des agents qu'il soit fait mention dans le rapport des projets et démarches développés par la direction générale dans les décennies précédentes. Les relectures et usages de l'histoire constituent souvent des enjeux stratégiques : ils le sont ici tout particulièrement dans la mesure où l'évocation des projets initiés par la DG V dans les années soixante en matière d'harmonisation des systèmes de protection sociale est ce qui permet aux auteurs du rapport d'inscrire la définition d'un modèle social dans la continuité des réflexions engagées dès l'origine de la construction européenne. Le terme d' " harmonisation » forgé dans cette période renvoyait ainsi à l'idée que dans la perspective du Marché Commun, il convenait de réduire, par le rapprochement des systèmes de protection sociale, les distorsions de concurrence que pourraient entraîner des régimes trop différents, et d'offrir, à des travailleurs européens susceptibles d'exercer dans tous les Etats membres, un niveau de protection identique. La réactivation de ces thèmes permet d'établir un parallèle avec la situation de l'élargissement en insistant notamment, comme dans les années soixante, sur les risques de dumping social qu'elle présente ${ }^{30}$. Comme l'illustre l'un des courriers adressés aux auteurs du rapport, cette référence est toutefois systématiquement contestée et n'apparaîtra plus dans la version définitive du document.

"Sur le chapitre 5/La sécurité sociale, il faut biffer la phrase: 'l'harmonisation des systèmes de sécurité sociale, d'abord tentée par la CEE'. La CEE n'a jamais essayé d'harmoniser les régimes de sécurité sociale car elle était consciente que cela s'avérerait comme une mission impossible et puis elle n'avait pas suffisamment de compétences pour le faire. " (Lettre d'un membre de la DG V, direction "sécurité sociale et programmes d'action sociale », en date de juillet 1997)

L'occultation des ambitions historiques du service s'explique pour partie par le malaise que suscite toujours, chez les eurofonctionnaires l'évocation de projets qui ont échoué et des conflits notamment politiques qu'ils ont suscités ${ }^{31}$. Mais le rejet de cette histoire indique plus

30 « Si les PECO devaient encore réduire la portée de leur système de protection sociale [...], il est possible qu'une pression croissante survienne pour substituer au système complet de protection sociale un simple filet de sécurité minimal, très éloigné des normes européennes actuelles et des objectifs de convergence en politique sociale. (...). Le risque de dumping social et d'exportation du chômage ne peut donc être négligé. " Commission des CE, DG IA (1996), Stratégie du secteur social, Version du 23 octobre 1996, 42p. \& 6 annexes, Bruxelles, octobre 1996, document interne et resté confidentiel, (p.29).

31 Voir sur ce point les réflexions de Marc Abélès et de ses co-auteures concernant le rapport au passé qui caractériserait l'administration communautaire. «Tout se passe comme si la Commission n'était pas en mesure de 
généralement que leur difficulté à penser l'existence d'un modèle social européen tient notamment au fait qu'elle repose sur des conceptions - du sens de l'intégration communautaire dans ce domaine, et du rôle que la Commission doit y jouer - auxquels il ne leur semble plus possible d'adhérer. Bien que les données recueillies ne permettent pas de l'étayer systématiquement, l'hypothèse est permise que ces postures sont d'autant plus marquées chez les fonctionnaires les plus jeunes. Comme l'évoque cet entretien avec un agent d'une trentaine d'année, dont la DG V est la première affectation :

"Avant, il y avait un peu un rêve au niveau européen d'avoir un beau jour un système harmonisé : c'est-à-dire la Communauté européenne faisant des directives et ainsi garantissant que tous les ouvriers qui se déplacent au sein de l'Union européenne n'ont aucune difficulté. Mais bon, comme soit il n'y a pas de base juridique dans le Traité pour faire quelque chose, soit il y a unanimité requise pour avoir une décision, et qu'en plus beaucoup d'Etats membres - dont l'Allemagne, et le Royaume-Uni-s'opposent, les gens de la Commission ont dit que, peut-être, ce n'est pas nécessaire de vouloir harmoniser, c'est même dangereux. " (Membre de la DG V, juin 1996, direction " sécurité sociale et programmes d'action sociale », Bruxelles)

La contestation de l'existence d'un " modèle social européen » à laquelle se livrent les agents interrogés repose par ailleurs sur deux types d'arguments. C'est d'abord l'idée de considérer les systèmes de protection sociale des Etats membres comme un ensemble relativement unifié et cohérent - et d'en dégager des critères objectifs à partir desquels évaluer les dispositifs existants dans les pays d'Europe centrale et orientale - qui est ici présentée comme irréaliste autant qu'illégitime. Alors que l'approche adoptée par la DG V jusqu'au début des années quatre-vingt dix avait consisté à souligner la convergence des politiques sociales nationales ${ }^{32}$, elles apparaissent désormais irréconciliables. Pour ces eurofonctionnaires, toute tentative de définir des principes communs serait condamnée à ne refléter qu'un point de vue partiel et partial. En outre, le tableau qu'ils dressent de l'évolution des dispositifs nationaux fait de ces dernier le reflet d'une histoire et d'une culture spécifiques, propres à chaque pays, l'expression d'une identité nationale que toute initiative au niveau européen reviendrait indirectement à menacer.

«Le problème, c'est qu'il n'y a pas de système de protection sociale européen parfait, il n'y a pas de benchmark, il n'y a pas de références. Parce qu'on voit bien que les systèmes que nous avons, les Quinze, sont très différents. Vraisemblablement, le système des Etats-Unis ou celui du Japon sont encore beaucoup plus différents. Mais quand même, il y a des différences considérables entre les systèmes que nous avons à l'intérieur de l'Union. Exemple : quelle est la façon optimale parfaite que nous avons d'organiser un fonds de pension? Le Royaume-Uni va dire qu'il faut mettre l'accent sur la capitalisation. Nous le savons. Les Allemands vont dire: 'Mais non, le système par contributions, c'est beaucoup mieux'. Qu'est-ce que la Commission peut dire? Alors, bon, chacun présente des avantages et des inconvénients, on ne peut jamais dire : 'il faut faire ça'. Alors, dans cette fonction de donner des conseils, nous avons une marge de manæuvre très réduite. (...) C'est un peu difficile parce que comment mesurer une protection sociale? Dans le domaine des monnaies, c'est facile, on a des taux de change, des choses

penser son propre rapport à l'histoire. Comme si elle était tiraillée entre deux extrêmes : soit exhiber le passé, mettre en spectacle le temps des pionniers dans une sorte de discours apologétique sur elle-même ; soit à l'inverse renvoyer aux oubliettes le souvenir de moments conflictuels, aussi proches soient-ils. » M. Abélès et alii, Approche anthropologique de la Commission, op. cit., (p.31).

32 Il s'agissait notamment de montrer, à travers le financement de travaux sur la comparaison des évolutions nationales, et l'organisation de rencontres entre experts des administrations nationales, que la coordination au niveau européen des politiques nationales était d'autant plus pertinente qu'un processus de convergence était déjà, naturellement, à l'œuvre. Voir par exemple : M. Ferrara, «Modèles de solidarité, divergences, convergences : perspectives pour l'Europe », Revue Suisse de Science Politique, 2(1), 1996. 
comme ça, mais pour la protection sociale c'est presque impossible d'écrire ça d'une façon objective, et de manière politiquement acceptable. (...) Et puis, quels critères utiliser pour comparer? C'est difficile parce que nous avons des statistiques, mais il y a toujours des questions comme : 'qu'est-ce que la pauvreté ?', 'qu'est-ce qu'un niveau de protection sociale ?' (...) Je crains que les différentes catégories ne soient trop différentes pour vraiment pouvoir les comparer. ( ) Non, il vaut mieux avoir une compétition des systèmes. Si nous avons des systèmes de protection sociale différents, ça, c'est bien, alors il y a une espèce de compétition des idées politiques. » (Membre de la DG V, direction « sécurité sociale et programmes d'action sociale », novembre 1996, Bruxelles)

Certains soulignent enfin, dans une perspective plus managériale, la trop grande charge administrative - tout au moins au regard des capacités de la Commission - qu'entraîneraient fatalement à plus ou moins long terme de telles initiatives.

"Aujourd'hui, je crois que ce serait vraiment difficile de gérer un système harmonisé de protection sociale en Europe, ce serait, à mon avis, beaucoup plus difficile que l'Union économique et monétaire, parce qu'après tout, dans une Union économique et monétaire, on a seulement des billets qui sont identiques, il n'y a pas d'administration, en dehors de cette banque centrale, mais c'est une administration relativement petite. Mais la protection sociale, hein, c'est un monstre. " (Membre de la DG V, direction "sécurité sociale et programmes d'action sociale », juin 1997, Bruxelles)

En soulignant l'illégitimité - historique, culturelle et même technique - de l'Union européenne et de son administration, ces eurofonctionnaires témoignent ainsi non seulement d'une révision à la baisse de leurs ambitions, mais aussi d'une forme de réappropriation des critiques auxquelles ils ont été régulièrement exposés. La nécessité de préserver les prérogatives du «niveau national », considéré comme le seul espace légitime de négociation et de mise en œuvre des politiques sociales est en effet une des principaux motifs de refus exprimés par les Etats membres hostiles au développement de compétences communautaires dans ce domaine ${ }^{33}$. Le débat qui s'est ouvert ces deux dernières années autour de la question du « modèle social européen » illustre du reste la constance de cette argumentation et son usage étendu, bien au delà de la rhétorique intergouvernementale de certains pays membres. C'est ce qu'évoque notamment la déclaration du vice-président de la Commission, le social démocrate allemand Günter Verheugen, dans les pages du journal Le Monde en septembre 2005 : «Chaque pays a ses traditions. Il est inutile d'essayer d'unifier nos systèmes sociaux. Dans chaque pays, on dépense à peu près la même chose en proportion dans le social, mais avec des méthodes différentes ${ }^{34}$. »

L'audience et l'autorité conquise par cette conception «nationalisée » des systèmes de protection sociale, insistant sur leur incompatibilité, fait ainsi obstacle à l'idée de modèle, entendu comme une construction théorique visant à subsumer la diversité de ces systèmes, à les représenter à partir d'un ensemble de caractéristiques communes. Mais c'est également la seconde signification du terme de " modèle », celle d'une solution idéale et susceptible d'être imitée, que les agents de la DG V semblent rejeter. Pour un certain nombre d'entre eux, en effet, le fait que les systèmes de protection sociale en vigueur dans les Etats membres puissent - malgré ou grâce à leur diversité - être érigés en exemple pour les pays d'Europe de l'Est n'a rien d'une évidence. A nouveau, ces discours semblent en outre offrir un écho singulier à la campagne, déployée en Europe de l'Est, par les organismes financiers internationaux comme la Banque Mondiale et le Fonds Monétaire International, pour convaincre les gouvernements

33 S. Leibfried, P. Pierson (dir.), Politiques sociales européennes : entre fragmentation et intégration, op. cit.

34 Le Monde, 2 septembre 2005. 
de ces pays de l'inefficacité des modèles de protection sociale ouest-européens ${ }^{35}$. Les dispositifs institutionnels expérimentés en Europe occidentale sont-ils valables? Les évaluations et bilans qu'il est possible de dresser de ces systèmes sont-ils suffisamment positifs pour qu'il soit crédible d'inciter les autres pays à s'en inspirer? Autant de questions qui affleurent dans les entretiens, évoquant une forme de découragement diffus, et témoignant plus généralement de l'absence d'une doctrine forte sur ces sujets jusque dans les services de la Commission qui en ont la responsabilité.

"Les deux grands sujets qui nous intéressent c'est tout de même l'emploi d'une part et la protection sociale, d'autre part. Bon, mais enfin, là, l'Ouest n'a pas tellement de leçons à donner puisqu'on est très mal parti, nous aussi. Bon, on est volontariste sur les questions d'emploi, la Conférence Intergouvernementale va accoucher d'un chapitre sur l'emploi, mais moi je suis un peu sceptique sur les capacités de l'Europe en matière d'emploi. (...) Alors pour moi, les politiques de l'emploi ça doit faire partie de l'acquis communautaire. Mais, c'est pas très facile comme exercice, de dire, de défendre que ça fait partie de l'acquis communautaire, parce que l'acquis communautaire en matière d'emploi, c'est quoi ? Dix millions de chômeurs? C'est de l'acquis également, l'acquis de quoi ? Où est l'acquis ?" (Membre de la DG V, direction « dialogue social », juin 1997, Bruxelles)

\section{Conclusion}

Les postures adoptées par ces agents témoignent ainsi des effets produits par le traitement des questions sociales sur la scène bruxelloise mais aussi, dans une certaine mesure, internationale. Sédimentées dans des dispositifs institutionnels qui contribuent largement à verrouiller les configurations existantes en conservant notamment un droit de veto à tout Etat membre désireux de ne pas voir les enjeux sociaux devenir européens, les stratégies politiques, juridiques et rhétoriques déployées par les adversaires de l'Europe sociale (d'autant plus nombreux que le débat européen agrège presque malgré eux opposants au social et opposants à sa communautarisation) informent aussi les pratiques et dispositions des membres de la Commission. Médiatisées par une culture d'institution qui érige en principes la défiance à l'égard du débat contradictoire, la nécessité et la valeur intrinsèque du compromis, l'occultation de la dimension politique du travail administratif, elles sont incorporées par les membres de la DG V : elles les conduisent ainsi à privilégier le repli sur la défense du droit existant, à renoncer à l'usage de certains arguments, et même à réécrire l'histoire de leurs origines.

Ce faisant, et c'est là l'essentiel, ceux que leurs prérogatives institutionnelles (via leur pouvoir d'initiative conféré à la Commission) et plus informelles (position de centralité dans

\footnotetext{
${ }^{35}$ Les réformes engagées dans les pays d'Europe centrale et orientale dans le cadre de leur transition politique et économique ont en effet suscité un investissement fort de ces organismes qui s'est traduit notamment par la provision d'expertise en matière de politiques sociales et l'inscription de conditions liées à leur réforme dans la plupart des prêts destinés à ces pays. Le discours de leurs experts confond dans une même critique le modèle communiste et un modèle dit «continental " - désignant, plus ou moins explicitement, les systèmes français, allemand, et ceux de l'Europe du Nord - dénonçant leur caractère désincitatif, leur crise financière et leur inefficacité en termes de redistribution aux plus pauvres. Il vise ainsi à promouvoir leurs propres modèles de politiques sociales (retraite par capitalisation, réduction de la protection sociale à l'assistance aux plus démunis, remise en cause du rôle des partenaires sociaux) auprès des responsables politiques est-européens et à influencer, à plus long terme, les débats communautaires. Fonds Monétaire International, Le dialogue de politique économique avec le FMI : la dimension sociale, Washington, FMI, 1995 ; B. Deacon, Globalization and social policy: the threat to equitable Welfare, Geneva, United Nation Research Institute for Social Development (UNRISD), 2000.
} 
le jeu européen, accès à l'expertise, maîtrise des outils et langage juridique) pouvaient prédisposer à faire avancer l'Europe sociale, participent ainsi à son non avènement. A l'instar des fonctionnaires de la culture décrits par Vincent Dubois, ils « réalisent par la faiblesse de leur investissement et les incertitudes de leurs pratiques la prophétie de leur illégitimité à intervenir ${ }^{36} \gg$ et celle de l'Europe sociale à se construire.

Dans cette perspective, l'exemple analysé ici nous amène à souscrire à l'hypothèse formulée par Didier Georgakakis et Marine de Lassalle concernant le « libéralisme » de la Commission, lequel s'apparente moins à un « libéralisme doctrinal » qu'à un « libéralisme de situation » lié notamment aux contraintes propres à cet espace institutionnel ${ }^{37}$. Nul besoin en effet de postuler l'adhésion des agents de la DG V à un discours politique explicitement fondé sur des valeurs et principes libéraux - que la plupart des entretiens conduits dans le cadre de cette enquête semblent du reste contredire - pour observer et comprendre qu'ils paraissent néanmoins se conformer à de tels principes.

C'est peut-être enfin dans cet apparent paradoxe qu'il faut chercher l'une des explications de la force avec laquelle « les canons économiques de la compétitivité et de la régulation » s'imposent et imprègnent l'action publique formulée au niveau européen. Ces " canons » trouvent en effet au sein même de la Commission des agents qui sont non seulement "disposés » à les suivre, mais qui contribuent aussi à en dénier publiquement l'influence. Les rapports de force, et certaines lectures politiques du monde, qui sont au principe du silence politique de l'Union européenne sur sa dimension sociale se reproduisent par l'intermédiaire de ces agents avec d'autant plus d'efficacité qu'ils sont ici largement occultés : c'est très rarement en référence explicite à ces contraintes politiques qui s'exercent sur eux, à l'extérieur et au sein même de l'institution que ces eurofonctionnaires motivent leurs positions. A travers les justifications qu'ils produisent, la définition de l'acquis restreinte à sa dimension juridique, et plus généralement l'impossible revendication d'un modèle social communautaire, s'imposent en prenant l'apparence du seul compromis démocratique possible, mais aussi du plus fidèle à l'héritage national et européen.

Liste des sigles utilisés :

PECO : pays d'Europe centrale et orientale

DG IA : direction générale de la Commission en charge des relations politiques extérieures, Europe et nouveaux Etats indépendants, politique étrangère et de sécurité commune, service extérieur ${ }^{38}$

DG II : direction générale en charge des affaires économiques et financières

DG V : direction générale en charge de l'emploi, des relations industrielles et des affaires sociales

DG XV : direction générale en charge du marché intérieur et des services financiers

\footnotetext{
36 V. Dubois, « L'art et l'Etat au début de la IIIème République, ou les conditions d'impossibilité de la mise en forme d'une politique », art. cit, (p.8).

37 Voir leur proposition d'article pour ce même numéro.

38 Les portefeuilles et leurs intitulés officiels ayant subi quelques modifications, on indique ici les appellations en cours au moment de l'enquête
} 